\title{
Contatti E CONTAGi, POESIa ITALiANa IN BRASILE: NUOVE TESTUALITÀ
}

\begin{abstract}
Patricia Peterle $*$
ABSTRACT: Tra la cultura italiana e quella brasiliana sono tanti i casi di nuove testualità o di "fenomeni di ibridizzazione". Per quel che concerne i rapporti letterari la mappatura della cartografia di questi contatti e contagi è complessa, producendo nuove riscritture a partire dalla letteratura italiana tradotta in Brasile.

PAROLE CHIAVE: contatti; poesia brasiliana; poesia italiana; traduzione letteraria

RESUMO: Entre a cultura italiana e a brasileira há muitos casos de novas textualidades ou "fenômenos de hibridização". No que diz respeito às relações literárias, o mapeamento da cartografia destes contatos e contágios é complexa, produzindo novas reescrituras a partir da literatura italiana traduzida no Brasil. PALAVRAS CHAVE: contatos; poesia brasileira; poesia italiana; tradução literária
\end{abstract}

ABSTRACT: Between the Italian and Brazilian cultures are so many cases of new textuality or "hybridization phenomena". As for the literary relations, mapping cartography of these contacts and contagions is complex, producing new rewrites from Italian literature translated in Brazil.

KEYWORDS: contacts; Brazilian poetry; Italian Poetry; literary translations

* Universidade Federal de Santa Catarina, Florianópolis (Brasil) - patriciapeterle@gmail.com

Processo n.2013/20971-0 Fundação de Amparo à Pesquisa do Estado de São Paulo (FAPESP)

DOI: http://dx.doi.org/10.11606/issn.2238-8281.v0i33p25-32 
el secolo della barbarie, come si sa, c'è una ripresa di Dante, in tutta la letteratura italiana e non solo, basti pensare a T.S. Eliot o a Jorge Luis Borges ${ }^{1}$. Per quel che riguarda il Brasile, accanto al non facile lavoro di traduzione dei tanti che si sono impegnati su questi versi, si possono ricordare Augusto e Haroldo de Campos, Cristiano Martins - in quella che è considerata oggi la miglior traduzione - e più recentemente Ítalo Eugenio Mauro, autore di un'edizione integrale, pubblicata nel 2011, dopo dodici intensi anni di lavoro ${ }^{2}$. O ancora, per continuare nella sfera dantesca, quando si leggono i versi del poeta brasiliano Carlos Drummond de Andrade, qui riprodotti nella traduzione di Antonio Tabucchi, non si può non ricordare l'indimenticabile incipit dell'Inferno.

Nel mezzo del cammino c'era una pietra

c'era una pietra nel mezzo del cammino

c'era una pietra

nel mezzo del cammino c'era una pietra.

1 Quest'articolo è parte di uno studio più ampio presentato al Convegno dell'Associazione Internazionale dei Professori di Italiano, realizzato presso l'Università di Bari, nel 2014.

2 Si vedano i saggi di Maria Teresa Arrigoni, in particolare: ARRIGONI, M.T. Em busca das obras de Dante em português no Brasil (1901-1950). In PETERLE, P. (org.). A literatura italiana no Brasil e a literatura brasileira na Itália. Tubarão: Copiart, 2011. 
Non mi scorderò mai di quell'avvenimento

nella vita delle mie rétine stanche.

Non mi scorderò che nel mezzo del cammino

c'è una pietra

c'era una pietra nel mezzo del cammino

nel mezzo del cammino c'era una pietra. (ANDRADE, 1987, p. 7)

In una specie d'ibridizzazione, i versi di Dante, letti da Drummond, in modo antropofagico, si trasformano in nuovi versi, nuovi suoni in una nuova lingua che è il portoghese. Ovviamente, molti altri poeti sono stati tradotti, e qui si potrebbero citare Carducci, Leopardi, il cui famoso L'infinito vede un gran ventaglio di versioni, che vanno dal già menzionato poeta concretista Haroldo de Campos a Henriqueta Lisboa, da Vinicius de Moraes a Marco Lucchesi e così via. Manzoni è un altro classico italiano che viene tradotto con una certa frequenza. L'opera alla quale si sono dedicati molti traduttori d'oltreoceano è senz'altro I promessi sposi, in un primo momento tradotta probabilmente dal francese presso i tipi della Garnier di Rio de Janeiro. Una curiosità è che Manzoni ha mantenuto uno stretto giro di corrispondenza con Dom Pedro II, imperatore brasiliano, che ha poi tradotto il "Cinque maggio" (Cf. CENNI, 2003, p. 86-87).

Tanti altri esempi potrebbero essere citati in questo studio, ma vorrei soffermarmi su un altro di quei casi che si potrebbero chiamare "fenomeni d'ibridizzazione" o nuove testualità, frutto di contatti e contagi. Nella complessa mappatura della cartografia della letteratura italiana tradotta in Brasile, ${ }^{3}$ un caso interessante è quello dei poeti Giuseppe Ungaretti e Ivo Barroso. Come si sa, a metà degli anni '30, Ungaretti si trasferisce in Brasile, con la famiglia, per inaugurare la prima cattedra d'italianistica nel paese sudamericano. Nel periodo del suo soggiorno brasiliano, ha la possibilità di scoprire tante cose, avere sentimenti nuovi - fra tutti, la perdita del figlio Antonietto - e confrontarsi con una cultura altra, straniera. Un rapporto, in effetti, fatto di molti

3 Per gli studi sulla letteratura italiana tradotta in Brasile, si fa riferimento a: PETERLE, P. (org.). A literatura italiana traduzida no Brasil e a literatura brasileira traduzida na Itália: sob o olhar da tradução. Tubarão: Copiart, 2011; PETERLE, P.; SANTURBANO, A.; WATAGHIN, L. (orgs.). Literatura italiana traduzida no Brasil 1900-1950. Rio de Janeiro: Comunità, 2013. Si vedano anche i due Dizionari Bibliografici: www.dlit.ufsc.br e www.usp.br/dlit. 
contatti e contrasti, ${ }^{4}$ ma che segna la sua vita e la sua poesia. Se qui parliamo della poesia italiana tradotta in Brasile, non si può non ricordare che, nel suo ritorno in Italia nei primi anni '40, Ungaretti sarà uno dei primi divulgatori della letteratura brasiliana, pubblicando delle traduzioni e scrivendo delle prefazioni, postfazioni e note. ${ }^{5}$

$\mathrm{Ma}$, a questo punto, ci si potrebbe chiedere che cosa c'entri Ungaretti con il poeta e traduttore Ivo Barroso, di cui adesso si parlerà, dal momento che Ivo Barroso non ha preso parte ai progetti editoriali in commercio riguardanti le traduzioni di Ungaretti. ${ }^{6}$ Se si pensa alle traduzioni di Ivo Barroso forse le più famose sono quelle dall'inglese, come i sonetti di Shakespeare - alcuni tradotti anche da Montale -, dei brani di Edgar Allan Poe e T.S. Eliot. Barroso ha inoltre curato l'edizione brasiliana delle poesie e prose di Charles Baudelaire, nonché tradotto André Malraux, André Gide, Georges Perec e Marguerite Yourcenar. Per quel che riguarda la letteratura italiana, oltre ad essere un grande lettore, Barroso si è dedicato a testi di Carlo Collodi, Italo Svevo, Italo Calvino, Umberto Eco, per arrivare infine, cosa non meno importante, al suo lavoro con le poesie di Eugenio Montale, Diario postumo, la cui traduzione esce nel 2000 - questo saggio non intende entrare nel merito del dibattito in corso sull' attribuzione o meno delle poesie riunite in questo volume all'autore degli Ossi di seppia.

La traduzione può essere vista come un proficuo esercizio di scrittura "per altri”, uno scrivere quindi non in proprio, che aiuta però ad avere una "pronuncia" propria, che pian piano si delinea. Infatti, Marco Lucchesi, poeta italo-brasiliano, che firma la presentazione della traduzione di Montale fatta da Barroso, sottolinea la capacità e la delicatezza con cui il poeta-traduttore brasiliano coglie gli "insights, in giustissime ricreazioni”. Nulla è scappato alla sua minuziosa traduzione: Barroso ha saputo operare il verso montaliano e la sua intensa maglia poetica. Come afferma ancora Lucchesi,

Non si può negare: la traduzione di poesia è una vera e propria prova della maturità letteraria di un paese. E Ivo Barroso - con la sua fine stampa da poeta - è nome

4 Cf.. PETERLE, P. Giuseppe Ungaretti: passagens e paragens. In MARSAL, M.H.; DINIZ, A. G.; CUSTODIO, R. De Faria C. (orgs.). Fluxos Migrantes. Vol. 1. Rio de Janeiro: Comunità, 2013, p. 100-113.

5 Cf. LANCIANI, G. (org.). Il Brasile di Ungaretti. In Quaderni di Libri e riviste D'italia, n. 52, Roma: Istituto Poligrafico e Zecca dello Stato, 2003.

6 I due volumi in Brasile con le traduzioni di G. Ungaretti sono: Daquela estrela à outra, a cura di Lucia Wataghin, traduzione Haroldo de Campo e Aurora F. Bernardini, São Paulo: Ateliê Editorial, 2003; Alegria, traduzione e note di Geraldo Holanda Cavalcanti, Rio de Janeiro: Record, 2003. 
cristallino. E ci offre ora, tramite la sua estetica puntuale e delicata, questa memorabile raccolta del grande poeta Eugenio Montale.(LUCCHESI, 2000, p. 13)

Dopo aver avuto modo di dedicarsi a Diario Postumo, che esce presso la Record (la stessa che ha pubblicato uno dei volumi di Ungaretti), in un'intervista del 2002 al periodico mensile di letteratura Rascunho, è lo stesso Barroso a dichiarare che da tempo si stava preparando alla traduzione dell'emblematico volume Ossi di Seppia (BARROSO, 2016). Tuttavia, la traduzione di queste poesie di Montale esce, sempre presso la Record, a firma di Renato Xavier.?

Non può essere una semplice coincidenza che, dopo il tanto lavorare con un'altra lingua, nel 2001 esca il terzo libro di poesia di Barroso. Il titolo di questa raccolta è paradigmatico per un poeta che è traduttore: La caccia virtuale (2001). Quale caccia? La caccia che non smette mai di cacciare? Sempre in cerca della sua preda? Sono alcune delle ipotesi che affiorano leggendo questo titolo enigmatico. $\mathrm{O}$ ancora, la preda può essere la parola; la parola che scivola - per usare un termine di Drummond - nel lavoro con il linguaggio poetico? Oppure le ceneri, un qualcosa che resta del lavoro come traduttore? L'esperienza rimasta e rielaborata dell'impossibilità/ necessità della traduzione? Dell'inquietudine e del lutto, per riprendere Paul Ricoeur? Questo resto, questo frammento, molte volte non percettibile, che non viene del tutto eliminato, è passibile di trasformazione, di diventare materia prima: dal fuoco alle ceneri e, potenzialità di queste ultime, dalle ceneri al fuoco. La tensione nella traduzione è forza creatrice e, quindi, passa a costruire e stabilire nuovi rapporti. Tradurre è, senza dubbio, costruire contatti ed essere contagiati.

La caccia virtuale di Ivo Barroso è un volume che raccoglie brani di vari momenti della sua vita e non presenta una preoccupazione di carattere cronologico. L'edizione non viene accompagnata da note o date che potrebbero fornire degli indizi al lettore un po' più attento. Come dice lo stesso Barroso, sempre nell'intervista già citata, "Non sempre è possibile lanciare luce sulla vita; alle volte è anche necessario convalidare l'eccesso di luce per far sì che le cose siano più visibili. Anche la poesia vive di ombre" (BARROSO, 2016, trad. nostra). Di fronte a queste parole, si potrebbe formulare una domanda: l'ombra qui sarebbe quella delle interlocuzioni con le traduzioni fatte durante vari anni, che ora vengono "a galla"? Se prima i riflettori illuminavano la faccia del traduttore e lasciavano in ombra un'altra, quella del poeta, ora il sentiero della luce è cambiato, illumina il viso del poeta e nasconde quello del traduttore.

7 MONTALE, E. Ossos de sépia. Trad. R. Xavier. Rio de Janeiro: Record, 2002. 
O si potrebbe addirittura affermare che ne illumina un'altra, in momenti non più così facili da distinguere? Certamente, queste due facce non sono bipartite, formano invece un unico corpo di chi parla, di questo io, traduttore-poeta/poeta-traduttore, che si inscrive?, quando scrive.

Tale ipotesi diviene ancora più interessante e inquietante quando si apre il libro e ci si rende conto che la prima poesia è un resto, cioè delle ceneri, dei fuochi, dei sentieri percorsi dal poeta-traduttore, una traccia che si dispiega; e in questo movimento del dispiegamento, l'autore mostra le sue innumerevoli pieghe e segni. Sulla pagina di Barroso, ci sono due soli versi, eppure due importanti segni, uniti da un titolo che li conferma, racchiudendo in sé tutto il componimento. Vediamo i due versi:

Alumbro-me de sombras

(BARROSO, 2001, p. 23)

Una volta letta questa poesia-frammento, musicale, sonora, quasi un flash di suoni e un'immagine, che fa vedere ed è accecata dalle ombre, ciò che resta della pagina è un grande spazio bianco. A questo punto è praticamente impossibile non pensare agli emblematici versi di Ungaretti in Allegria (1931):

\section{Mattina}

Santa Maria La Longa il 26 gennaio 1917

Mi illumino

d'immenso

(UNGARETTI, 1969, p. 65)

Ora si può togliere la maschera e inserire il titolo dato da Barroso alla composizione: "A Ungaretti”. Ora tutto può sembrare più chiaro. Un omaggio? Un tentativo di traduzione senza un buon esito? Un insuccesso? Non importa, queste parole, questi versi appartengono ormai anche a Barroso, fanno parte della sua lingua. Sempre nell'intervista concessa a Rascunho, quando gli si chiede di questa poesia, risponde: 
Quando provavo a tradurre il celebre verso di Ungaretti, mi sono reso conto che era impossibile mantenere in portoghese ciò che è stato detto nel modo in cui è stato detto. In italiano il verbo è sdrucciolo (illumino) e le doppie hanno un valore fonico, oltre a quello visuale. La funzione di quelle "mm" offre al poema la forma fisica della sua immensità. La soluzione è stata quella di conservare la forma e modificare il contenuto, fino al punto di sovvertirlo, affinché si ottenesse un simile effetto. Però, a questo punto, non era più una traduzione, ma sì una poesia propria, nonostante mi fossi appropriato di una forma altrui: 50\% traduzione, $50 \%$ originale. Così, l'ho dedicato a Ungaretti e incluso nel libro. (BARROSO, 2016, trad. nostra)

L'importante qui è notare come nella lettura fatta da Barroso della poesia di Ungaretti, si stabilisca, pian piano, uno spazio tra Ungaretti e Barroso, mediato dal testo, ma in nessun momento limitato da esso. Il tentativo di traduzione è forse fallito, ma questo fa parte del babelico universo della lingua. La traduzione di questi versi di Ungaretti è rimasta viva nella tensione della sua impossibilità/necessità, ma alla fine si è generata, o si è operato qualcosa di nuovo che, insieme agli altri frammenti, sopravvive ancora nel titolo del volume, La caccia virtuale. Secondo Eduardo Portella, che firma la prefazione, le poesie del libro sono anche frutto di un "convivere che è vivere con", se si pensa alle possibilità ed ai sentieri percorsi sulla pagina in bianco e davanti a un'origine, la cui esistenza è valida solo nell'intervallo tra l'avvicinamento e la distanza. Le soluzioni poetiche, riprendendo ancora Portella, vanno trovate nelle complesse tessiture delle soluzioni trovate per i sonetti di Shakespeare, nelle ricreazioni di T.S. Eliot, nelle incorporazioni di un Rimbaud e concentrate nelle meditazioni di Montale. Nessuno può passare impunemente per queste letture $\mathrm{e}$, in questo caso specifico, c'è addirittura la forte componente di ciò che è stato chiamato fenomeno d'ibridizzazione.

Per cui, in questo senso, la traduzione è un movimento ampio e complesso, fondamentale tra le culture, necessario alla vita, alla sopravvivenza di un testo, che pian piano si dispiega in altri e in altre letture. In effetti, per Walter Benjamin (1995), è addirittura nella traduzione che la vita dell'originale raggiunge, in modo sempre rinnovato, il suo più ampio dispiegamento: dal testo da tradurre al testo tradotto, potendosi anche tornare dal testo tradotto, sempre con qualcosa di diverso, a quello da cui si è partiti (Fortleben). 


\section{Riferimenti bibliografici}

ANDRADE, C. Drummond de. Sentimento del mondo. Torino: Einaudi, 1987.

BARROSO, I. A caça virtual e outros poemas. Rio de Janeiro: Record, 2001.

BARROSO, I. “A busca da metáfora real”.https://gavetadoivo.wordpress.com/2010/09/24/a-buscada-metafora-real/ Acesso em 15/06/2016.

BENJAMIN, W. Angelus novus. Torino: Einaudi, 1995.

CENNI, F. Italianos no Brasil: andiamo in 'Merica'. São Paulo: EDUSP, 2003.

DRUMMOND, C. Il sentimento del mondo. Trad. Antonio Tabucchi. Torino: Einaudi: 1987.

LUCCHESI, M. Prefácio. In MONTALE, E. Diário póstumo. Trad., introd. e notas de I. Barroso. Rio de Janeiro: Record, 2000.

UNGARETTI, G. Tutte le poesie. Milano: Mondadori,1969.

Recebido em 13/03/2017

Aprovado em 15/06/2017 